\title{
Measurement of step surface contour based on variable sampling phase shift interference phase extraction algorithm based on selective sampling
}

\section{Songsong Zhang}

Guizhou University,Key labortory of Advanced Manufacturing Technology of Ministry of Education Haisong Huang ( $\square$ hshuang@gzu.edu.cn )

Guizhou University,Keylaboratory of Advanced Manufacturing Technology of Ministry of Education https://orcid.org/0000-0002-3750-6537

\section{Qiaoqiao Xiong}

Department of Mecjanical an Manufacturing Engineering,Faculty of Engineering,University Putra Malaysia

\section{Research}

Keywords: Phase shift, Optical accuracy, Phase extraction, Iteration

Posted Date: August 17th, 2020

DOI: https://doi.org/10.21203/rs.3.rs-56303/v1

License: (c) (i) This work is licensed under a Creative Commons Attribution 4.0 International License. Read Full License 


\title{
Measurement of step surface contour based on variable sampling phase shift interference phase extraction algorithm based on selective sampling
}

\author{
Songsong Zhang1, Haisong Huang ${ }^{1 *}$, Qiaoqiao Xiong ${ }^{2,3}$ \\ 1. Guizhou University, Key laboratory of Advanced Manufacturing Technology of Ministry of Education, Huaxi, \\ Guiyang, China, 550025; 2. Department of Mechanical and Electronic Engineering, Guizhou Communications \\ Polytechnic, Guiyang 551400, China; 3.Department of Mechanical and Manufacturing Engineering, Faculty of \\ Engineering, Universiti Putra Malaysia, Serdang, Selangor 43400, Malaysia
}

\begin{abstract}
Variable frequency phase shift interferometry is widely applied in optical precision measurement, with the accuracy of phase extraction's direct impact on that of phase shift interferometry. In the variable-frequency phaseshift interferometry, the commonly used phase-shifting devices are prone to phase shift errors, because the ordinary equal-step phase extraction algorithm, which can be merely used to measure simple and smooth surface, influences the accuracy of phase extraction resulting in measuring error, and causes inefficiency led by the long time the iterative process lasts for when applied in complex stepped surfaces measurement. As a sort of step-by-step phase-shifting phase extraction algorithm based on selective sampling is used to measure the step surface contour, the interference image is firstly sampled at equal intervals to reduce the iterative calculation, and in view of the fact that the phase calibration of the test system is not required in this algorithm, the measured phase is given by using the alternating iterative method despite the unknown phase and unknown phase shift amount. The phase extraction accuracy and iteration time among traditional iterative algorithm, four-step phase shift algorithm and the variable phase shift phase interpolation algorithm based on selective sampling are compared in the simulation and experiment. It is shown that the variable frequency phase shifting interference phase extraction algorithm based on selective sampling has shorter operation time, less error and higher accuracy than traditional iterative algorithm in measuring complex step surface.
\end{abstract}

Keywords: Phase shift, Optical accuracy, Phase extraction, Iteration.

* Haisong Huang, E-mail: hshuang@gzu.edu.cn

\section{Introduction}

The interferometric method to measure the high-accuracy surface shape was first carried out in the

1960s. With the rapid development of interferometry technology, phase-shifted interferometry (phaseshifting interferometry, PSI), the primary presentative of interferometry technology, becomes one of the main methods in modern measurement of optics surface shape. From the early phase-shift algorithm including the Hariharan algorithm and the Schwider algorithm to the current frequency-conversion phase-shift algorithm including the iterative algorithm and the principal element analysis method, we can come to a conclusion that the research of the frequency 
conversion phase shift algorithm has become the trend of modern phase-shift interferometry research[1]. Although the principal element analysis (PCA) and Advanced iterative algorithm (AIA) can be used to recover the phase information from a large number of phase-shifted interferogram efficiently[2-5], a series of error sources and the complexity of measured surface do exist in the actual measuring process[6,7]. And the traditional phase-shift interference algorithm exposes some drawbacks, such as the effect of noise and phase shift error on phase extraction precision of stochastic phase-shift algorithm[8], the influence of the background intensity and modulation system on phase-shift algorithm, and slow rate of operation as well as low precision of the result $[3,9,10]$.

In order to solve the above drawbacks, Yang Mu, Hou Lizhou and others proposed a stochastic phase-shifted phase solution method based on least squares iteration, whereas running computing time will be increased obviously and phase extraction accuracy will mostly be lowered when interference fringe graphs with lots of pixels are available to be dealt with in the iterative operation period[3,11,12]. In this paper, the iterative algorithm is used to solve the phase, and it is improved and deeply researched, and a high efficient and accurate phase extraction algorithm is proposed, which differs from the traditional method in the study of phase extraction with random sampling to simplify operation and improve accuracy[13-16]. In the measurement process, the algorithm selectively samples the original interference fringe image, which ensures that all pixel points of the iterative operation are valid, thus lessening the amount of data[17-19]. Besides, in the case of unknown phase shifts and phases, the algorithm can determine the phase shift and phase simultaneously through constant iterative computing[15], and achieve higher accuracy in measuring complex step surface profile due to the insensitivity to the phase shift error.The reliability of the algorithm is verified by simulation experiment and experimental data[18,19]. 


\section{Basic principle}

\section{1 basic principle of frequency conversion phase shift interference}

In the frequency conversion phase shift interferometry, the assumption is that the background light is $I_{0}(x, y), \gamma_{0}(x, y)$ is the modulation, $h(x, y)$ is the length of the interference cavity, and the light intensity expression of the t-step phase shift is:

$$
I_{t}(x, y)=I_{o}(x, y)\left\{1+\gamma_{o}(x, y) \cos \left[\frac{4 \pi h(x, y)}{\lambda_{t}}\right]\right\}
$$

The $\lambda_{t}=\lambda_{0}+t \Delta \lambda$ represents the test wavelength value corresponding to the t-step phase shift, the $\lambda_{0}$ is the wavelength tunable semiconductor laser starting output wavelength (that is, the center wavelength), $\Delta \lambda$ is the wavelength phase shifting step, (1) can be approximated to:

$$
\begin{gathered}
I_{t}(x, y)=I_{o}(x, y)\left\{1+\gamma_{o}(x, y) \cos \left[\frac{4 \pi h(x, y)}{\lambda_{t}}-\frac{4 \pi h(x, y)}{\lambda_{o}^{2}} * t * \Delta \lambda\right]\right\} \\
\varphi_{i j}=\frac{4 \pi h(x, y)}{\lambda_{t}} \text { is the measured phase, } \delta_{k}=-\frac{4 \pi h(x, y)}{\lambda_{0}^{2}} * t * \Delta \lambda \text { is the amount of phase shift }
\end{gathered}
$$

in step $\mathrm{t}$.

It can be seen from equation (2) that in the variable-frequency phase-shift interferometry, the phase shift of the phase shift in the $\mathrm{t}$-th step is determined by the joint length and the wavelength step. In the measurement, in order to make the phase shift amount a certain value under different interference cavity lengths, phase calibration must be performed[20]. When the iterative algorithm is used to solve the measured phase, it can be determined by continuous iteration when the phase shift amount and phase are unknown, and the phase shift amount and the measured phase can be determined at the same time. Measurement accuracy, eliminating the impact of calibration error on test accuracy. 


\subsection{Frequency-shifted phase-shift interferometric phase extraction algorithm based on selection} sampling

In phase-shifted interferometry, if the phase shift $\delta_{k}$ can be determined, then the K-phase-shifted interference image can be expressed as:

$$
\begin{aligned}
& I_{k}(i, j)=a(i, j)+\gamma_{o}(i, j) \cos \left[\Phi(i, j)+\delta_{k}\right] \\
& k=0,1,2 \ldots k-1 ;
\end{aligned}
$$

In (3), $\mathrm{K}$ is the total number of phase shifts; $(i, j)$ is the pixel coordinates; $I_{k}(i, j)$ is the intensity distribution of the interference fringe pattern; $a(i, j)$ is the background intensity; $\gamma_{0}(i, j)$ indicates the degree of modulation; $\Phi(i, j)$ is the phase value to be solved; $\delta_{k}$ is the amount of phase shift per time, where $\delta_{0}=0$. In the formula (3), $I_{k}(i, j)$ is a known quantity, but $a(i, j), \gamma_{0}(i, j), \Phi(i, j)$ are unknown quantities.

Expanding (3) can take the following form:

$$
I_{k}(i, j)=C_{0}(i, j)+C_{1}(i, j)+C_{0}(i, j) \cos \delta_{k}+C_{2}(i, j) \sin \Phi(i, j)
$$

In the formu $C_{0}(i, j)=a(i, j) ; C_{1}(i, j)=\gamma_{0}(i, j) \cos \Phi(i, j) ; C_{2}(i, j)=-\gamma_{0}(i, j) \sin \Phi(i, j)$. According to the principle of least squares, the residual function is obtained by using the formula (4):

$$
E_{k}(i, j)=I_{k}(i, j)-\left[C_{0}(i, j)+C_{1}(i, j) \cos \delta_{k}+C_{2}(i, j) \sin \delta_{k}\right]
$$

To solve the optimal estimate of $C_{0}(i, j), C_{1}(i, j), C_{2}(i, j)$, we need to make the square sum of (5) and take the minimum value. Further acquisition of the phase value To be measured:

$$
\Phi(i, j)=\arctan \frac{-C_{2}(i, j)}{C_{1}(i, j)}
$$

The true phase shift amount during the phase shift can be expressed as the nominal phase shift plus the phase shift error value. The first iteration can make the initial phase shift error zero. 


$$
\hat{\delta}_{k}=\delta_{k}+\varepsilon_{k}
$$

Real interferogram intensity distribution:

$$
\hat{I}_{k}(i, j)=C_{0}(i, j)+C_{1}(i, j) \cos \left(\delta_{k}+\varepsilon_{k}\right)+C_{2}(i, j) \sin \left(\delta_{k}+\varepsilon_{k}\right)
$$

According to the above algorithm, a new $\hat{C}(i, j)=\left[\widehat{\mathrm{C}}_{0}(i, j), \hat{C}_{1}(i, j), \widehat{\mathrm{C}}_{2}(i, j)\right]^{T}$ can be obtained. Substituting the phase shift amount and the phase shift error into the formula, the residual function is as follows:

$$
\hat{E}_{k}(i, j)=\hat{I}_{k}(i, j)-\left[\hat{C}_{0}(i, j)+\hat{C}_{1}(i, j) \times \cos \delta_{k}+\hat{C}_{2}(i, j) \times \sin \delta_{k}\right]
$$

The phase shift error value in the formula is an unknown quantity[21-23]. Similarly, the phase shift error value can be solved by the least squares method. A partial derivative is obtained for each phase shift error amount $\varepsilon_{k}$, and the result is zero. The phase shift error value $\varepsilon_{k}$ can be

obtained by solving the equation. At this time, the phase shift amount $\widehat{\delta}_{k}=\delta_{k}+\varepsilon_{k}$ is updated to perform the next iteration operation. The threshold condition $\mathrm{T}$ is set according to the phase shift error value $\varepsilon_{k}$, and the iteration is terminated when the $\varepsilon_{k}$ change amount is less than $\mathrm{T}$. At this point, an accurate phase shift amount can be obtained[21,23].

\section{Simulation experiment based on selective sampling}

Use initial cap for first word in title or for proper nouns. Use lowercase following colon. Title should not begin with an article or contain the words "first," "new" or "novel." This paper simulates four interference fringe patterns with a pixel resolution of $450 \mathrm{pix} \times 450 \mathrm{pix}$. The contrast of a region in the interference image is reduced, and the low reflectance sample region in the actual measurement is simulated; and the salt and pepper noise with a density of 0.002 and the Gaussian 
noise with a standard deviation of 15 are added to the entire image to simulate the interference image noise. The interference diagram is shown in Figure. 1.

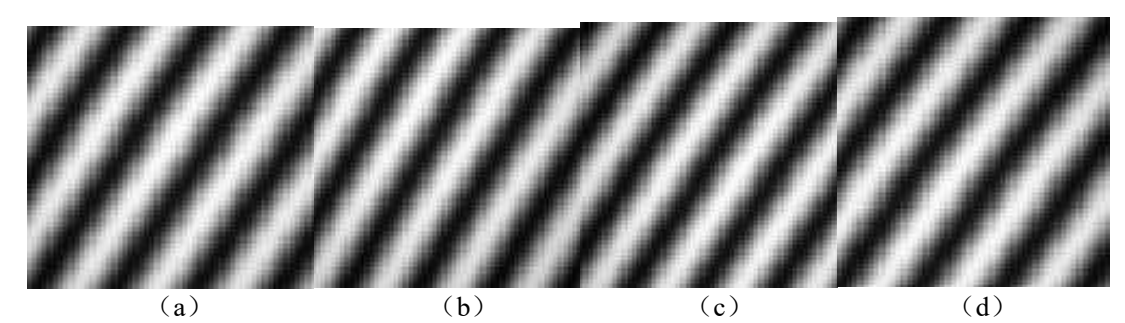

Fig. 1 Four simulated interference fringe images

The true value of the phase shift between the simulated interference fringe images is $\left[0^{\circ}, 101^{\circ}\right.$, $\left.191^{\circ}, 301^{\circ}\right] \cdot\left[0^{\circ}, 100^{\circ}, 195^{\circ}, 300^{\circ}\right]$ was calculated as the initial phase shift amount. The root mean square error (RMSE) between the result and the true phase shift value is used as a criterion for determining the iterative error value.

$$
R M S E=\sqrt{\frac{\sum_{i=1}^{4}\left(X_{o b j, i}-X_{t r u e, i}\right)^{2}}{4}}
$$

The original image is first sampled every d pixel points. Then the sampled points are brought into the iterative algorithm, the four-step phase shift algorithm and the improved algorithm for iterative calculation[25]. The images after equal interval sampling are filtered out at low quality points, and a reasonable threshold $\mathrm{T}$ is selected to divide the four interferences. In the fringe image, the point where the maximum value of the gray value of the pixel at the same position is smaller than the threshold $\mathrm{T}$ is filtered, and the pixel whose image value is always low in the image[26-28]. The threshold $\mathrm{T}=80$ is determined according to the pixel value of the dark interference fringe. At this time, the filtering ratio is $20 \%$, indicating that most of the sampling pixels still participate in the iterative operation after filtering, and the relationship between the sampling interval and the running time and the sampling interval and the error value are as shown in Figure. 2 and Figure. 3. 


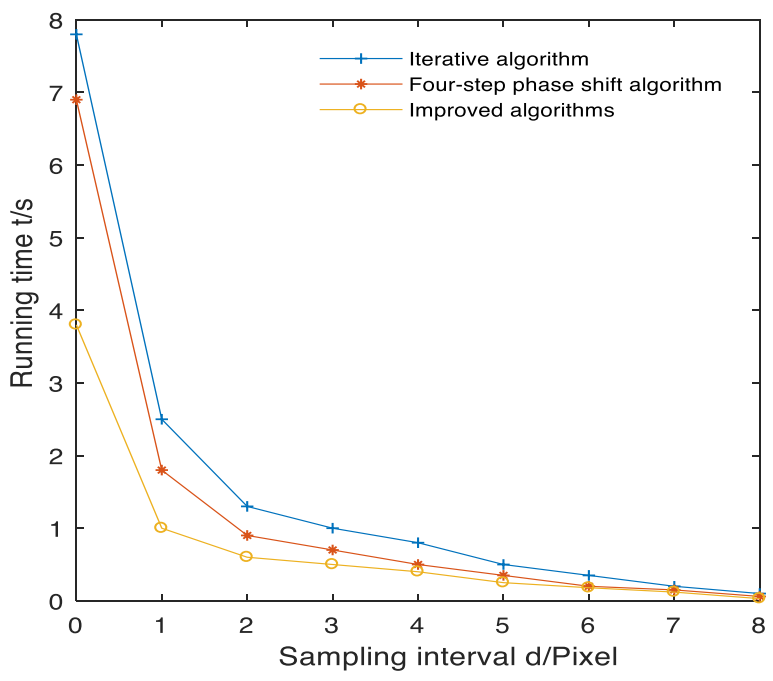

Fig. 2 Comparison of the running time of the three algorithms with equal interval sampling

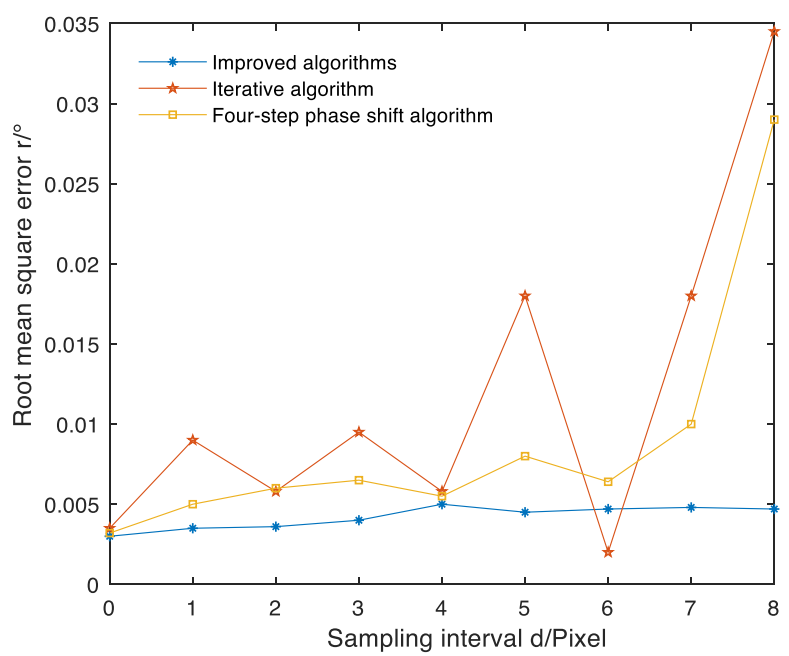

Fig. 3 Comparison of root mean square error of three algorithms for equal interval sampling

It can be seen from the simulation results that the iteration time of the equally spaced samples of the three algorithms is gradually reduced as the sampling interval increases, whereas the iteration time that improved algorithm spends at the same sampling interval is shortest. At the same time, the error values of the iterative algorithm and the four-step phase shift algorithm vary greatly with the sampling interval, and the error value is also large, while the variation of the error value of the improved algorithm is relatively stable and the error value is small. Comprehensively, the 
improved algorithm can effectively reduce the time of iterative calculation, and can keep the accuracy of algorithm at a high degree.

\section{Measurement experiment and analysis}

\subsection{Experimental system construction}

The optical path diagram of the measurement system we use is shown in Figure. 4. The system consists of a projector, an area array camera, a lens, a moving displacement platform, and a computer. A CCD camera is used to take a picture above the reference plane to obtain the surface contour of the object. Information and sync to the PC side. The image resolution of the camera is $768 \times 576$ pixels, and the actual size of the object corresponding to the width of the captured image is $100 \mathrm{~mm}$. By adjusting the distance between the lens and the projector, the scattered light field projected by the projector is projected through the lens and projected onto the object to be tested in parallel, and the projection angle of incidence is $30^{\circ}[25]$.

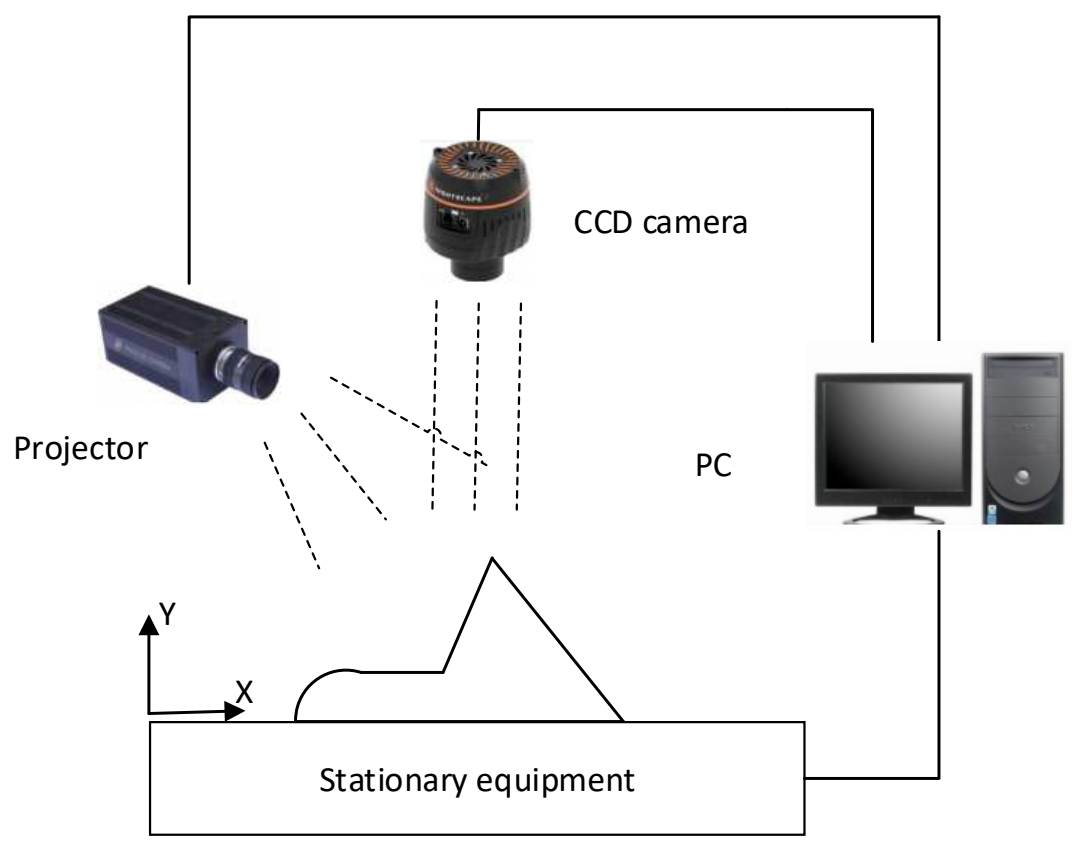

Fig. 4 Test system light path diagram 


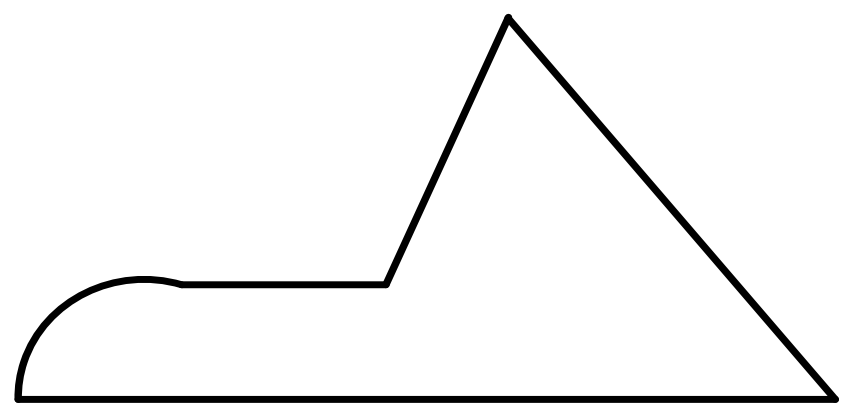

Fig. 5 measured surface section

The measured surface shape of the experiment uses a complex measured surface whose front end is a stepped surface with a smooth curved back end, as shown in Figure.5. In this way, we compare the variable frequency phase shift phase extraction algorithm based on selective sampling. Whether it is more accurate when measuring the step surface, the experiment includes the following steps:

(1) The phase-shifted interferograms with different phase shifts are obtained from the measured surface, and both have a random error with a mean of 0 and a variance of 0.01 ;

(2) Solving the measured phase by using an iterative algorithm, a four-step phase shift algorithm, and a variable frequency phase shift phase extraction algorithm based on selective sampling; (3) Unwrapping the package phase to obtain the measured surface for experimental analysis.

\subsection{Interferogram with phase shift amount of arbitrary value}

Let the phase shift step be an arbitrary value. The phase shift amount and the theoretical phase shift amount obtained by the above iterative method are as shown in Table 1, and the number of iterations is 65 times. 
Table 1 Phase shift by interaction method and the theoretical phase shift (arbitrary phase shift)

\begin{tabular}{|c|c|c|c|c|}
\hline Step & $\mathbf{1}$ & $\mathbf{2}$ & $\mathbf{3}$ & $\mathbf{4}$ \\
\hline $\begin{array}{c}\text { Theoretical phase } \\
\text { shift/rad }\end{array}$ & 0.9323154 & 2.0123545 & 4.1532456 & 5.2231458 \\
\hline $\begin{array}{c}\text { Phase shift by iteration } \\
\text { method/rad }\end{array}$ & 0.9323142 & 2.0123520 & 4.1532446 & 5.2231419 \\
\hline
\end{tabular}

From the above results, the simulated wave front diagram of Figure.6 (a) and the phase diagram of the envelope of Figure 6 (b) are obtained. It is found that the phase shift magnitude obtained by the iterative method is compared with the theoretical phase shift magnitude, and the error is $10^{\wedge}(-$ 5) .The order of magnitude indicates that the iterative algorithm is used to solve the phase shift with high precision, which can reduce the measurement error introduced by the step error during interferometry $[8,15]$.

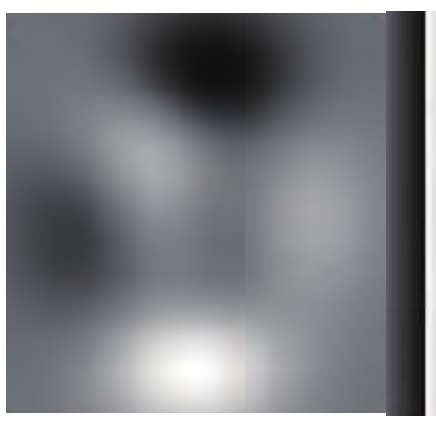

(a)

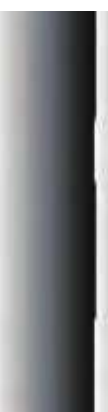

(b)

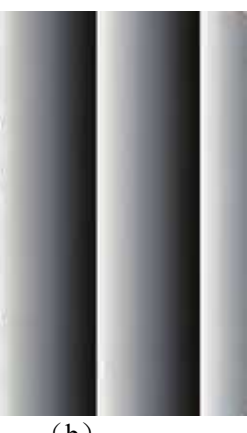

)

Fig. 6 (a) simulated wavefront map (b) wrapped phase map

\subsection{Iterative algorithm with phase shift of $\pi / 2$}

The iterative algorithm is used to solve the measured surface, and the phase shift and theoretical phase shift are as shown in Table 2. The number of iterations is 37.

Table 2 Phase shift by interaction method and the theoretical phase shift (arbitrary phase shift)

\begin{tabular}{|c|c|c|c|}
\hline Step & $\mathbf{1}$ & $\mathbf{2}$ & $\mathbf{3}$ \\
\hline Theoretical phase shift/rad & 1.560756 & 3.141456 & 4.712354 \\
\hline Phase shift by iteration method/rad & 1.560742 & 3.141425 & 4.712313 \\
\hline
\end{tabular}


The shape of the surface to be measured obtained after unwrapping and fitting is shown in Figure 7(a) and Figure 7(b) is the surface cross section of the surface to be measured.

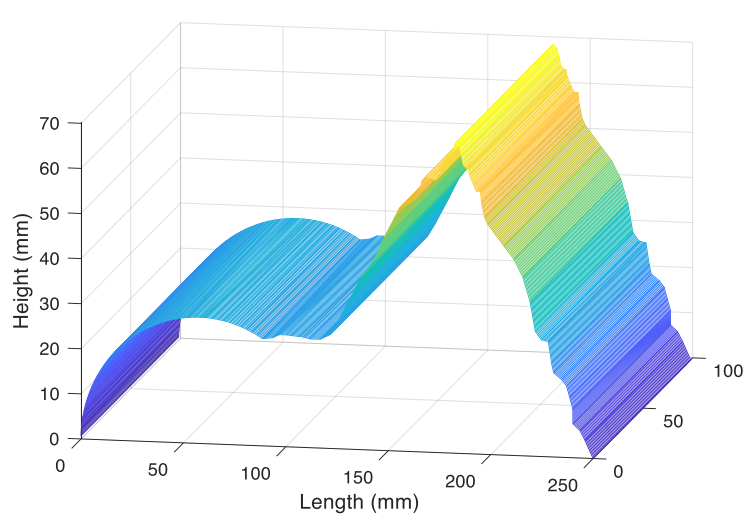

(a)The measured surface shape of the iterative method

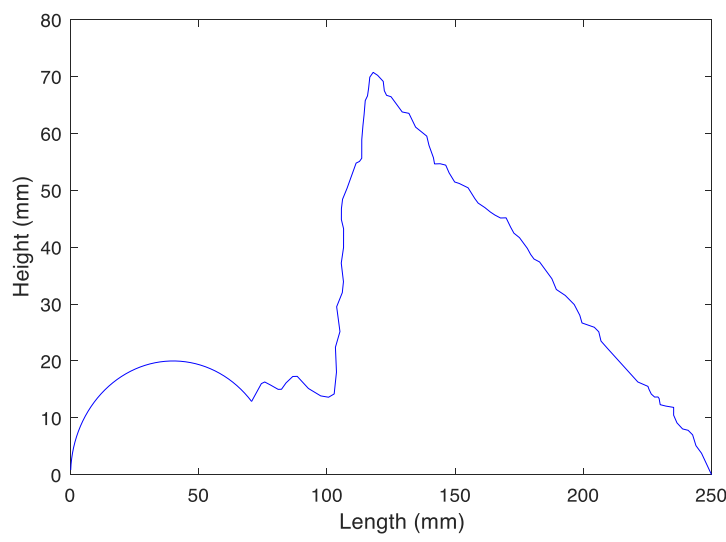

(b)The measured surface profile of the iterative method

Fig. 7 Results of the iterative algorithm measurement

\subsection{Four-step phase shift algorithm with phase shift of $\pi / 2$}

Using a four-step phase shift algorithm (four steps of phase shift, each phase shift is $\pi / 2$ ), four phase shifting interferograms with phase shift steps of $\pi / 2$ rad are acquired, and the four-step phase shift algorithm is used to test The phase solution formula $\varphi=\arctan \left(\frac{I_{4}-I_{2}}{I_{1}-I_{3}}\right)$, the phase shift amount and the theoretical phase shift amount are as shown in Table 3, and the number of iterations is 37 times.

Table 3 Phase shift by interation method and the theoretical phase shift(arbitrary phase shift)

\begin{tabular}{|l|l|l|l|}
\hline Step & 1 & 2 & 3 \\
\hline Theoretical phase shift/rad & 2.1325135 & 4.1536589 & 6.3254756 \\
\hline Phase shift by iteration method/rad & 2.1325125 & 4.1536545 & 6.3254742 \\
\hline
\end{tabular}

The shape of the surface to be measured obtained after unwrapping and fitting is shown in Figure $8(\mathrm{a})$, and Figure $8(\mathrm{~b})$ is the surface cross section of the surface to be measured. 

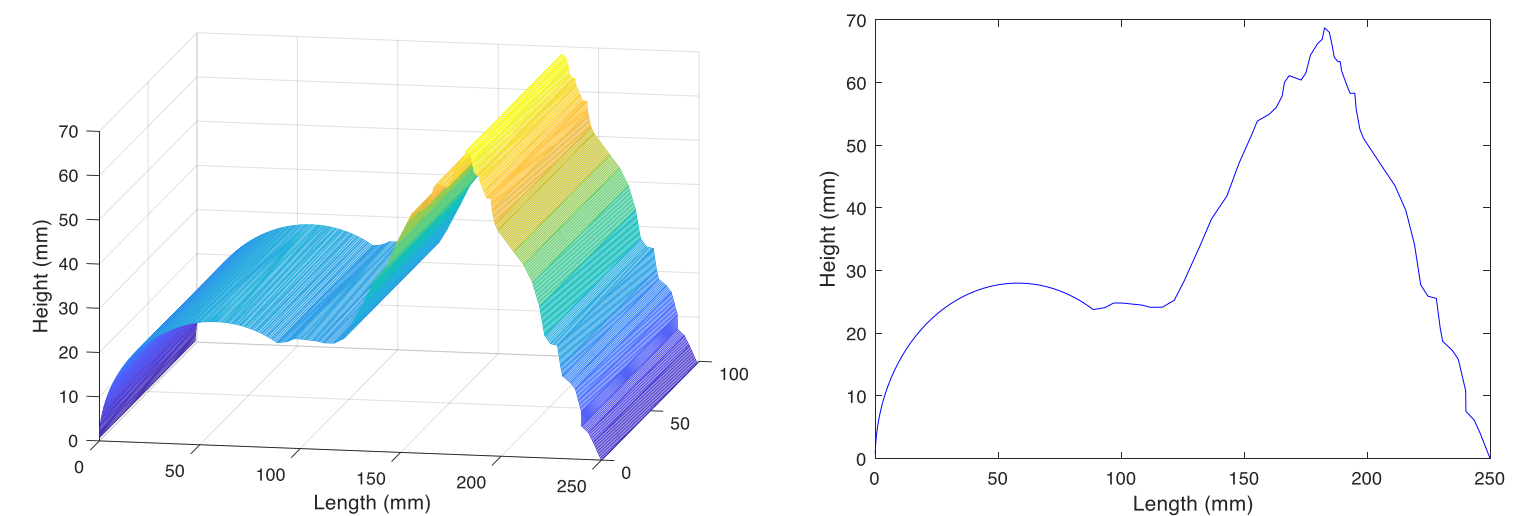

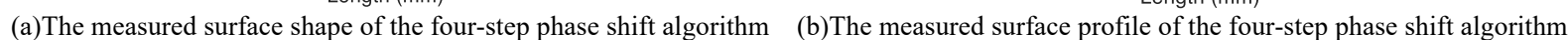
Fig. 8 Results of the iterative algorithm measurement

\subsection{Frequency-shifted phase-shift phase extraction algorithm based on selective sampling for} solving measured phase

The iterative-based phase extraction algorithm tends to be less accurate and time-consuming, and the efficiency is not high. It can be seen from the analysis of the iterative algorithm and the fourstep phase shift algorithm that the number of pixels in the whole graph substituted into the calculation is huge, leading to iteration. The efficiency is low. This paper proposes a method based on selective sampling for phase shift phase shifting. Firstly, the original image is equally spaced, and the sampling interval is a, that is, one pixel is sampled every a pixel; equal interval sampling can ensure The sampling point can represent the original interferogram to the greatest extent. At this time, the calculation method of the phase shifter error can be used according to the actual measurement process. When the phase shift error is small, a five-step phase shift fast phase extraction algorithm can be used. When the amount error is large, the maximum pixel value of the $\mathrm{K}$ interferogram can be directly used to represent the contrast, and the iterative calculation is performed.

Five interference fringes were collected in the experiment, and the true value of the phase shift between the interference fringe images was $\left[0^{\circ}, 76^{\circ}, 160^{\circ}, 230^{\circ}, 321^{\circ}\right] .\left[0^{\circ}, 80^{\circ}, 160^{\circ}, 240^{\circ}, 320^{\circ}\right]$ 
is taken as the initial phase shift amount into the algorithm, and then the original image is sampled every other pixel point, and then the sampled points are brought into The algorithm performs an iterative operation, and finally the profile of the measured surface obtained by unwrapping and fitting and the cross-sectional view of the measured surface shape are as shown in Figure 9(a) and Figure 9(b).
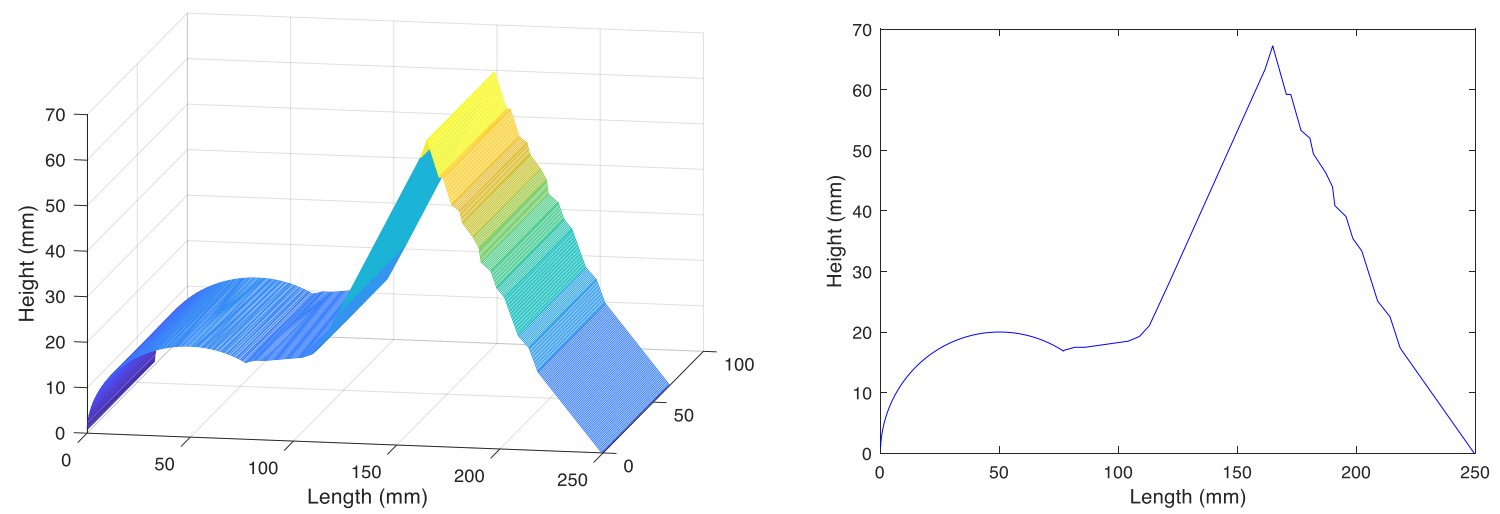

(a)The measured shape surface solved by the variable frequency phase shift phase extraction algorithm based on the selected sampling (b)Sectional view of the measured shape solved by the variable frequency phase shift phase extraction algorithm based on the selected sampling

Fig. 9 Results measured by a variable-phase phase shift phase extraction algorithm based on selected samples

\subsection{Analysis of results}

From the above three sets of experiments, from Figure 7. (a) and (b), Figure 8 (a) and (b) and Figure 9 (a) and (b), iterative algorithm, four-step phase shift algorithm and based on Selecting the sampled phase-shift phase-shifting algorithm to solve the shape of the wrapped phase, the above three algorithms can obtain the higher-precision measurement pattern. In the surface step of the measured surface, the iterative algorithm and the four-step phase shift algorithm are fitted to obtain a larger surface error value, and the measurement accuracy is lower, but when using the variable phase shift phase extraction algorithm based on selective sampling It can be seen from Figure 9 (a) and (b) that the surface error level obtained after fitting is kept at a relatively low level, and the 
maximum iteration error value is only about $0.1^{\circ}$, which has a high Measurement accuracy, and running time is shorter than the other two algorithms.

\section{Conclusions}

Phase-shifted interferometry has been widely used in 3D scanning modeling, image restoration, and accurate measurements. In actual measurements, the accuracy of phase result has a great impact on that of subsequent phase to be worked out. The fact that the accurate calibration of phase shift is hard to accomplish brings about phase shift errors inevitably, thus affecting the measurement accuracy. The experimental result shows that when the traditional iterative algorithm and the four-step phase shift algorithm are used to measure simple and smooth surface, the phase shift error has little effect on the measurement result with a high accuracy, whereas they are used to measure complex step surface, the measurement result has great influence with a apparently low accuracy./ The measurement result is not much affected by the phase shift error with a high accuracy when iterative algorithm and the four-step phase shift algorithm are used to measure simple and smooth surface, whereas it is greatly influenced with a low accuracy when used to measure complex step surface.

In the measurement of the variable phase shift phase extraction algorithm based on selective sampling, the method is used to solve the measured phase without phase calibration of the test system, and the measurement is not sensitive to the phase shift error. The variable phase shift phase extraction algorithm based on selective sampling can obtain the actual phase shift amount by accurate iteration and simultaneously sample the original image at equal intervals, after which it can perform sampling selectively, reducing the iterative calculation amount and ensuring the accuracy of iterative result. After verifying the efficiency and accuracy of algorithms by simulation and measurement experiments, we come to a conclusion that the algorithm proposed in this paper 
has a more scope application, not only to the simple and smooth surfaces, but also to complex step surfaces. When the algorithm mentioned before is used to measure complex step surfaces, a high accuracy and short running time can be reached. As a result, such algorithm can be used in actual measurements.

\section{Acknowledgments}

Not applicable.

\section{Authors' contributions}

All authors contributed equally in compiling this review. All authors read and approved the final manuscript.

\section{Funding}

This research was supported by the National Natural Science Foundation of China and the Guizhou Provincial Science and Technology Project. We gratefully acknowledge the support of Key laboratory of Advanced Manufacturing Technology with the donation of the Experimental equipment used for this research. Special thanks to all authors of the paper for their contributions to this work. The Authors acknowledge funding by: National Natural Science Foundation of China under Grant Nos.51865004, Key Project of Guizhou Province Science and Technology (JZ[2014]2001), Guizhou Provincial Nature Science Foundation (J-[2015]2043), Guizhou University Graduate Innovation Foundation (YLG 2016025), Guizhou Province Science and Technology Major Special Project（[2017]3004）.

\section{Availability of data and materials}

Upon request to the authors. 


\section{Ethics approval and consent to participate}

Not applicable.

\section{Competing interests}

The authors declare that they have no competing interests.

\section{Author details}

${ }^{1}$ Guizhou University, Key laboratory of Advanced Manufacturing Technology of Ministry of Education, Huaxi, Guiyang, China, 550025.

${ }^{2}$ Department of Mechanical and Electronic Engineering, Guizhou Communications Polytechnic, Guiyang 551400, China.

${ }^{3}$ Department of Mechanical and Manufacturing Engineering, Faculty of Engineering, Universiti Putra Malaysia, Serdang, Selangor 43400, Malaysia

\section{References}

1. Lenk L, Mitschunas B , Sinzinger S . Zoom systems with tuneable lenses and linear lens movements[J]. Journal of the European Optical Society Rapid Publications, 2019, 15(1):9.

2. Marcus Vinícius Rezende Júnior, José Eduardo Silveira Leal, Pires R R, et al. Traceability for measurements carried out on incremental step loading equipment[J]. Journal of the Brazilian Society of Mechanical ences and Engineering, 2019, 41(8):1-14.

3. Lu F, Wu C, Yang J . Three-dimensional shape measurement of complex surfaces based on optimized dithering fringe patterns[J]. Journal of the European Optical Society Rapid Publications, $2018,14(1)$ 
4. Rodriguezruiz, A., Teuwen, J., Vreemann, S., Bouwman, R. W., Van, R. E., \& Karssemeijer, N., et al. (2017). New reconstruction algorithm for digital breast tomosynthesis: better image quality for humans and computers. Acta Radiologica, 284185117748487. [CrossRef]

5. Xiao, D., Li, X., Liu, S. J., \& Wang, Q. H. (2018). Encryption and display of multiple-image information using computer-generated holography with modified gs iterative algorithm. Optics Communications, 410, 488-495. [CrossRef]

6. Milankovic, I. L., Mijailovic, N. V., Filipovic, N. D., \& Peulic, A. S. (2017). Acceleration of image segmentation algorithm for (breast) mammogram images using high-performance reconfigurable dataflow computers. Computational and Mathematical Methods in Medicine,2017,(2017-5-22), 2017(4), 7909282.

7. Guo, K., Jiang, J., \& Xu, Y. (2017). Approximation of stochastic quasi-periodic responses of limit cycles in non-equilibrium systems under periodic excitations and weak fluctuations. Entropy, 19(6), 280.

8. Wang, M., Du, G., Zhou, C., Si, S., Li, X. L., \& Lei, Z., et al. (2017). Enhanced two-frequency phaseshifting method based on generalized phase-shifting algorithm. Journal of Modern Optics, 64(16), 112. [CrossRef]

9. Zhang, X. X., Wang, Y. M., Huang, S. J., Gao, N., \& Zhang, Z. H. (2017). A two-step phase-shifting algorithm for phase calculation. Acta Photonica Sinica, 46(3).

10. Li, X., Zhao, S., Pan, S., Zhu, Z., Qu, K., \& Lin, T. (2017). Generation of a frequency-quadrupled phase-coded signal using optical carrier phase shifting and balanced detection. Appl Opt, 56(4), 11511156.

11. Peng, K., Cao, Y., Wu, Y., Chen, C., \& Wan, Y. (2017). A dual-frequency online pmp method with phase-shifting parallel to moving direction of measured object. Optics Communications, 383, 491499. [CrossRef]

12. Li, J., Zhong, L., Liu, S., Zhou, Y., Xu, J., \& Tian, J., et al. (2017). An advanced phase retrieval algorithm in n-step phase-shifting interferometry with unknown phase shifts. Sci Rep, 7, 44307. 
13. Cui, B., Chen, T., Wang, J., \& Yao, K. (2018). Calibration of wavefront phase image using phaseshifting iteration algorithm in quadriwave lateral shearing interferometry. Journal of the Korean Physical Society, 72(3), 359-365. [CrossRef]

14. Phuc, P. H., Rhee, H. G., \& Ghim, Y. S. (2018). Erratum: denoising phase unwrapping algorithm for precise phase shifting interferometry. Journal of the Korean Physical Society, 72(11), 1412-1412.

15. Cascavita, K. L., Bleyer, J., Chateau, X., \& Ern, A. (2018). Hybrid discretization methods with adaptive yield surface detection for bingham pipe flows. Journal of Scientific Computing(1), 1-20.

[CrossRef]

16. Zhou, R. Q. (2018). Application of on-line surface detection system in aluminum strip. World Nonferrous Metals.

17. Han, Y., Wang, Z., Li, D., Guo, Q., \& Liu, G. (2018). Low-complexity iterative detection algorithm for massive data communication in iiot. IEEE Access, PP(99), 1-1.

18. Bhavani, M., Reddy, K. T., \& Varma, P. S. (2018). An iterative genetic algorithm based source code plagiarism detection approach using ncrr similarity measure. Journal of Theoretical \& Applied Information Technology, 96(3), 543-554. [CrossRef]

19. Zhao, D., Jianyong, L. I., Cheng, W., \& Wen, W. (2018). Multi-fault feature detection of rolling element bearing by an iterative generalized demodulation algorithm under time-varying rotational speed. Journal of Vibration \& Shock. [CrossRef]

20. Zhao, S., Shen, B., \& Yang, F. (2017). Low complexity hybrid iterative algorithm based signal detection in massive mimo system. Telecommunications Science.

21. Tay, T. F., \& Chang, C. H. (2016). A non-iterative multiple residue digit error detection and correction algorithm in rrns. IEEE Transactions on Computers, 65(2), 396-408. [CrossRef]

22. Tozoni, D. C., Rezende, P. J. D., \& Souza, C. C. D. (2016). Algorithm 966: a practical iterative algorithm for the art gallery problem using integer linear programming. Acm Transactions on Mathematical Software, 43(2), 1-27. 
23. Yao, Y., Chen, K., Lin, Y., \& Zhou, C. (2017). Regression analysis with response-selective sampling. Statistica Sinica, 27(4). [CrossRef]

24. Liang, F., Jin, I. H., Song, Q., \& Liu, J. S. (2016). An adaptive exchange algorithm for sampling from distributions with intractable normalizing constants. Journal of the American Statistical Association, 111(513), 00-00.

25. Watters, K. E., Yu, A. M., Strobel, E. J., Settle, A. H., \& Lucks, J. B. (2016). Characterizing rna structures in vitro and in vivo with selective 2'-hydroxyl acylation analyzed by primer extension sequencing (shape-seq). Methods, 103(9), 34-48. [CrossRef]

26. Li, Y. (2017). High-accuracy brillouin frequency shift measurement system based on stimulated brillouin scattering phase shift. Optical Engineering, 56(5), 056102.

27. Cui, P., Li, S., Wang, Q., Gao, Q., Cui, J., \& Zhang, H. (2016). Harmonic current suppression of an amb rotor system at variable rotation speed based on multiple phase-shift notch filters. IEEE Transactions on Industrial Electronics, 63(11), 6962-6969.

28. Kano, H., Iseda, A., Ohenoja, K., \& Niskanen, I. (2016). Refractive index measurement of nanoparticles by immersion refractometry based on a surface plasmon resonance sensor. Chemical Physics Letters, 654, 72-75.

29. Kawai D, Aoki S, Doi T, et al. I=2\$\$ \$pi $\backslash$ pi $\$$ scattering phase shift from the HAL QCD method with the LapH smearing[J]. Progress of Theoretical and Experimental Physics, 2017, 2018(4).

30. Irfan S, Ahmed A, Park J H, et al. Current-Sensorless Power-Decoupling Phase-Shift Dual-HalfBridge Converter for DC-AC Power Conversion Systems Without Electrolytic Capacitor[J]. IEEE Transactions on Power Electronics, 2017, 32(5):10-3622.

31. Li K, Yuan L, Zhao Z, et al. Fault-Tolerant Control of MMC with Hot Reserved Submodules Based on Carrier Phase Shift Modulation[J]. IEEE Transactions on Power Electronics, 2017, 32(9):6778-6791.

32. Jesús Ernesto Arias-González, Fung T, Seymour R M, et al. A coral-algal phase shift in Mesoamerica not driven by changes in herbivorous fish abundance[J]. PLoS ONE, 2017, 12(4). 
33. Sonnen K F , Lauschke V M, Uraji J . Modulation of Phase Shift between Wnt and Notch Signaling Oscillations Controls Mesoderm Segmentation.[J]. Cell, 2018, 172(5):1079-1090.e12.

34. Penati T, Koukouloyannis V, Sansottera M, et al. On the nonexistence of degenerate phase-shift multibreathers in a zigzag Klein-Gordon model[J]. 2018.

35. Yakovleva T V . Determining the phase shift of quasiharmonic signals through envelope analysis[J]. Computer Optics, 2017, 41(6):950-956.

36. Ishida E, Miura K, Shoji Y, et al. Amorphous-Si waveguide on a garnet magneto-optical isolator with a TE mode nonreciprocal phase shift[J]. Optics Express, 2017, 25(1):452.

37. Li B . Round-robin differential-phase-shift quantum key distribution in wavelength-multiplexed fiber channel[C]// Fourth International Conference on Optical and Photonics Engineering. International Society for Optics and Photonics, 2017.

38. Yu P, Ma J, Wu T, et al. 8 phase-shift-keying optical millimeter-wave signal generation by electro-optical phase modulator-based photonic frequency quintupling[J]. Optical Engineering, 2019, 58.

39. Zhang B, Sun J, Li G, et al. Differential phase-shift keying heterodyne coherent detection with local oscillation enhancement[J]. Optical Engineering, 2018, 57.

40. Zhang D, Hao S, Zhao Q, et al. Differential phase-shift keying and channel equalization in free space optical communication system[J]. Optical Engineering, 2018, 57.

41. Maksimova L A, Ryabukho P V, Mysina N Y, et al. Determination of subpixel microdisplacements of speckle structure using the phase shift of spatial spectrum field[J]. Technical Physics, 2017, 62(8):1284-1287.

42. Ney M, Abdulhalim I, Ney M, et al. Combining three wavelength illumination and parallel phase shift interferometry for high-speed high-resolution and real-time motion tracking and 3D imaging (Conference Presentation)[C]// Novel Optical Systems Design \& Optimization XX. 2017. 
43. Jin L, Liu B, Duan S . ZVS operation range analysis of three-level dual active bridge DC-DC converter with phase-shift control[C]// 2017 IEEE Applied Power Electronics Conference and Exposition (APEC). IEEE, 2017.

44. Alanazi M D, Elbaksawi O, Abofard A E M , et al. Current doubler rectifier for arc welding machines with a phase-shift ZVS three-level DC-DC converter[C]// 2017 2nd International Conference on System Reliability and Safety (ICSRS). IEEE, 2017.

45. Fang L, Shi Z, Cheng X, et al. Geometrically tunable Fabry-Perot filters based on reflection phase shift of high contrast gratings[C]// Selected Papers of the Chinese Society for Optical Engineering Conferences held October and November 2016. International Society for Optics and Photonics, 2017.

\section{$\underline{\text { Caption List }}$}

Fig. 1 Four simulated interference fringe images.

Fig. 2 Four simulated interference fringe images.

Fig. 3 Four simulated interference fringe images.

Fig. 4 Test system light path diagram.

Fig. 5 measured surface section measured surface section.

Fig. 6 (a) simulated wavefront map (b) wrapped phase map.

Fig. 7 Results of the iterative algorithm measurement.

Fig. 8 Results of the iterative algorithm measurement.

Fig. 9 Results measured by a variable-phase phase shift phase extraction algorithm based on selected samples.

Table 1 Phase shift by interaction method and the theoretical phase shift (arbitrary phase shift).

Table 2 Phase shift by interaction method and the theoretical phase shift (arbitrary phase shift). 
Table 3 Phase shift by interation method and the theoretical phase shift(arbitrary phase shift). 
Figures

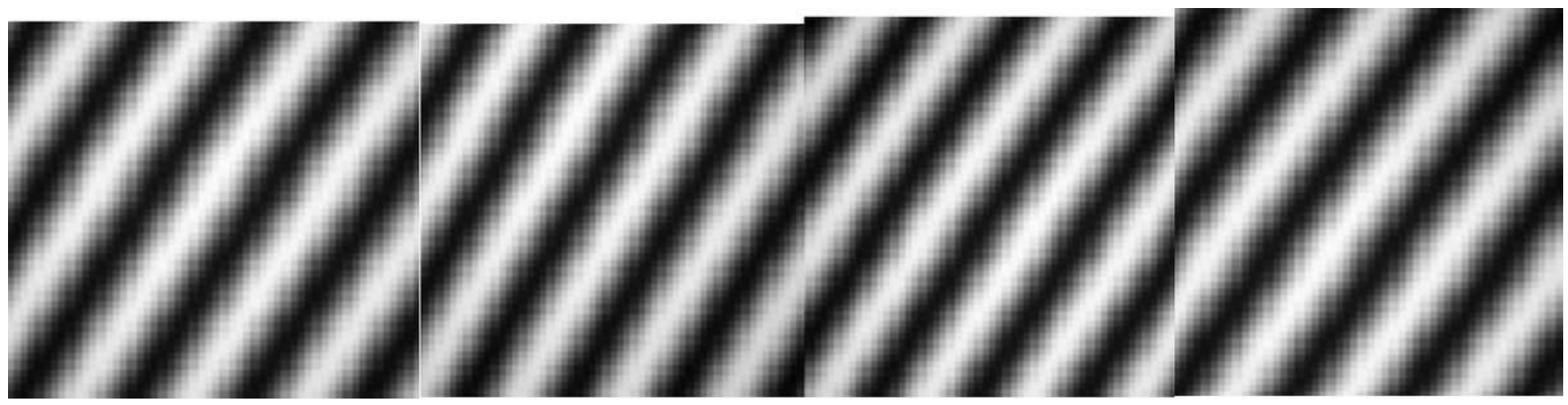

\section{Figure 1}

Four simulated interference fringe images

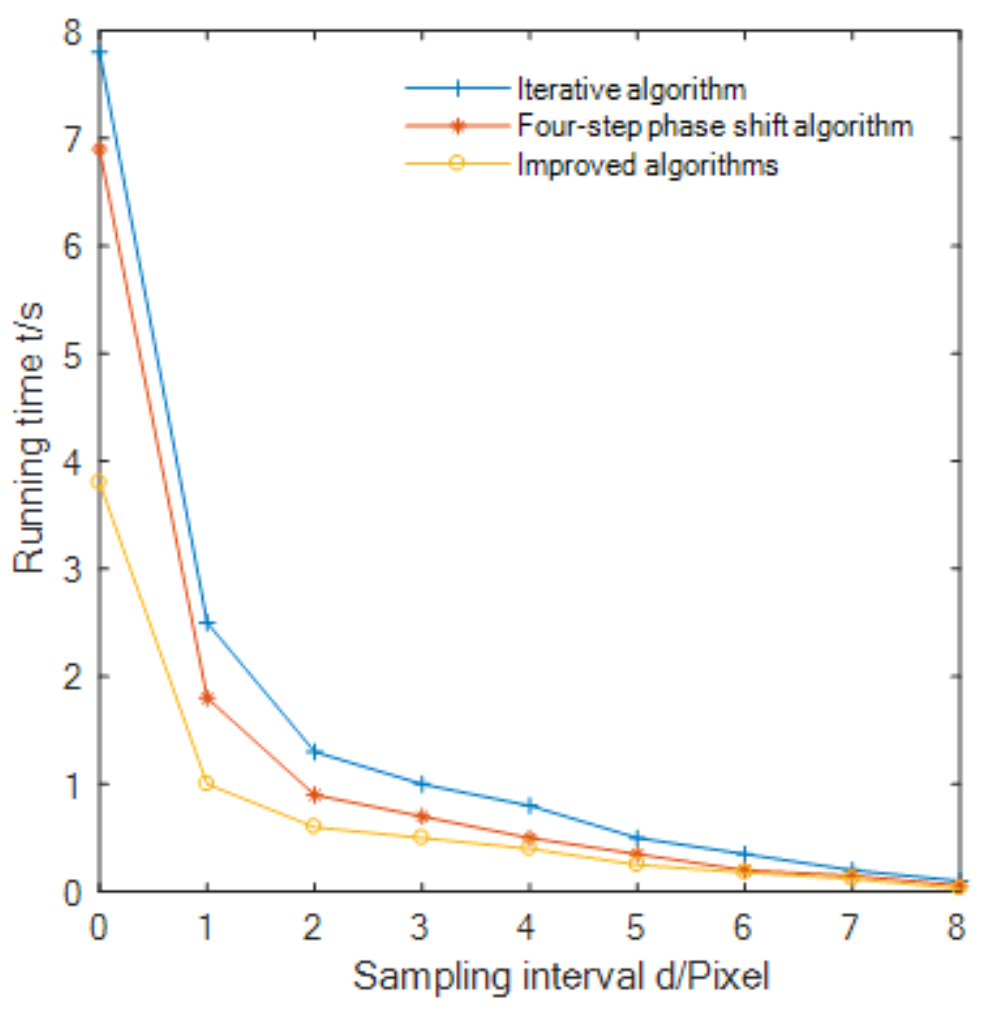

Figure 2

Comparison of the running time of the three algorithms with equal interval sampling 


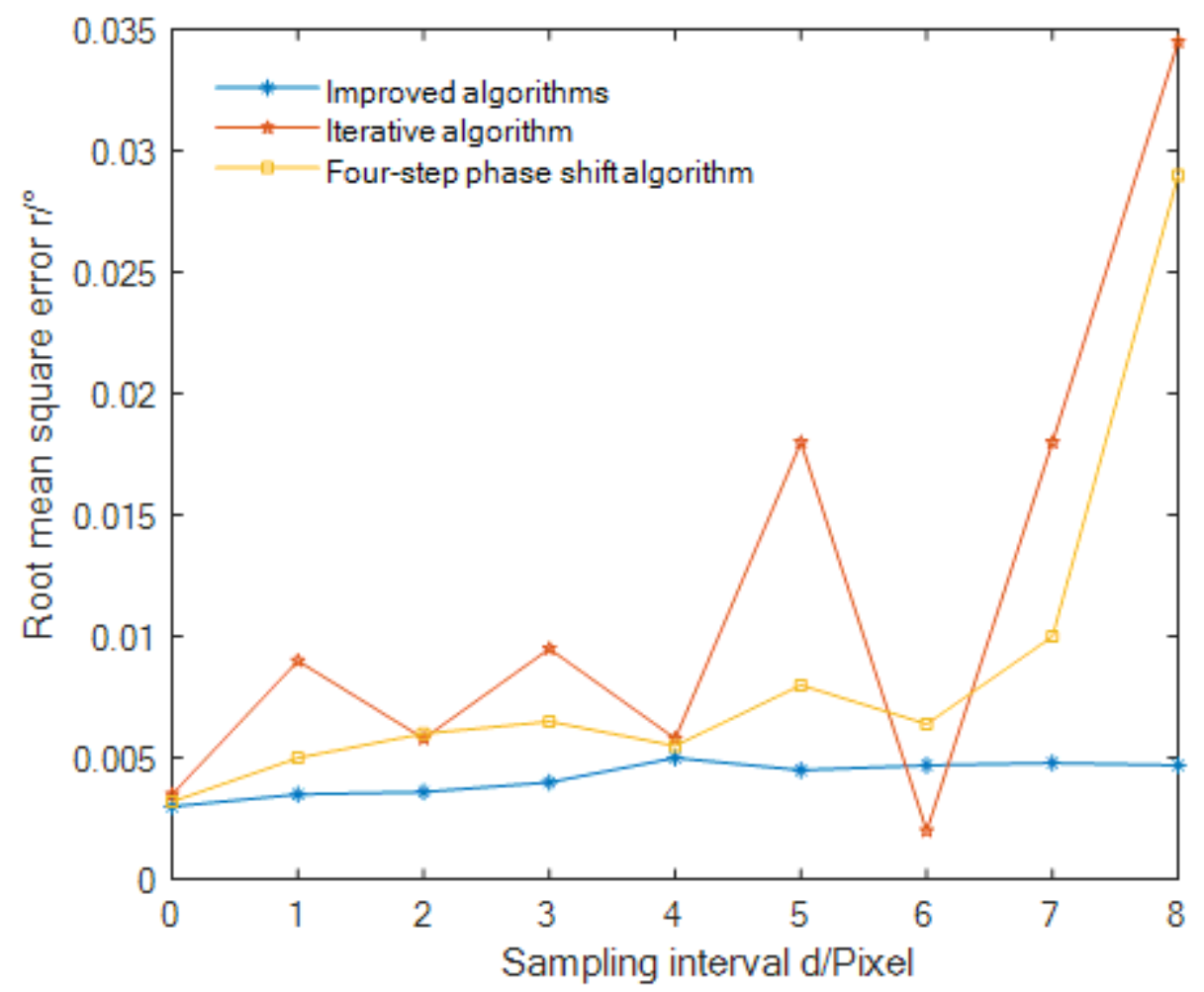

Figure 3

Comparison of root mean square error of three algorithms for equal interval sampling

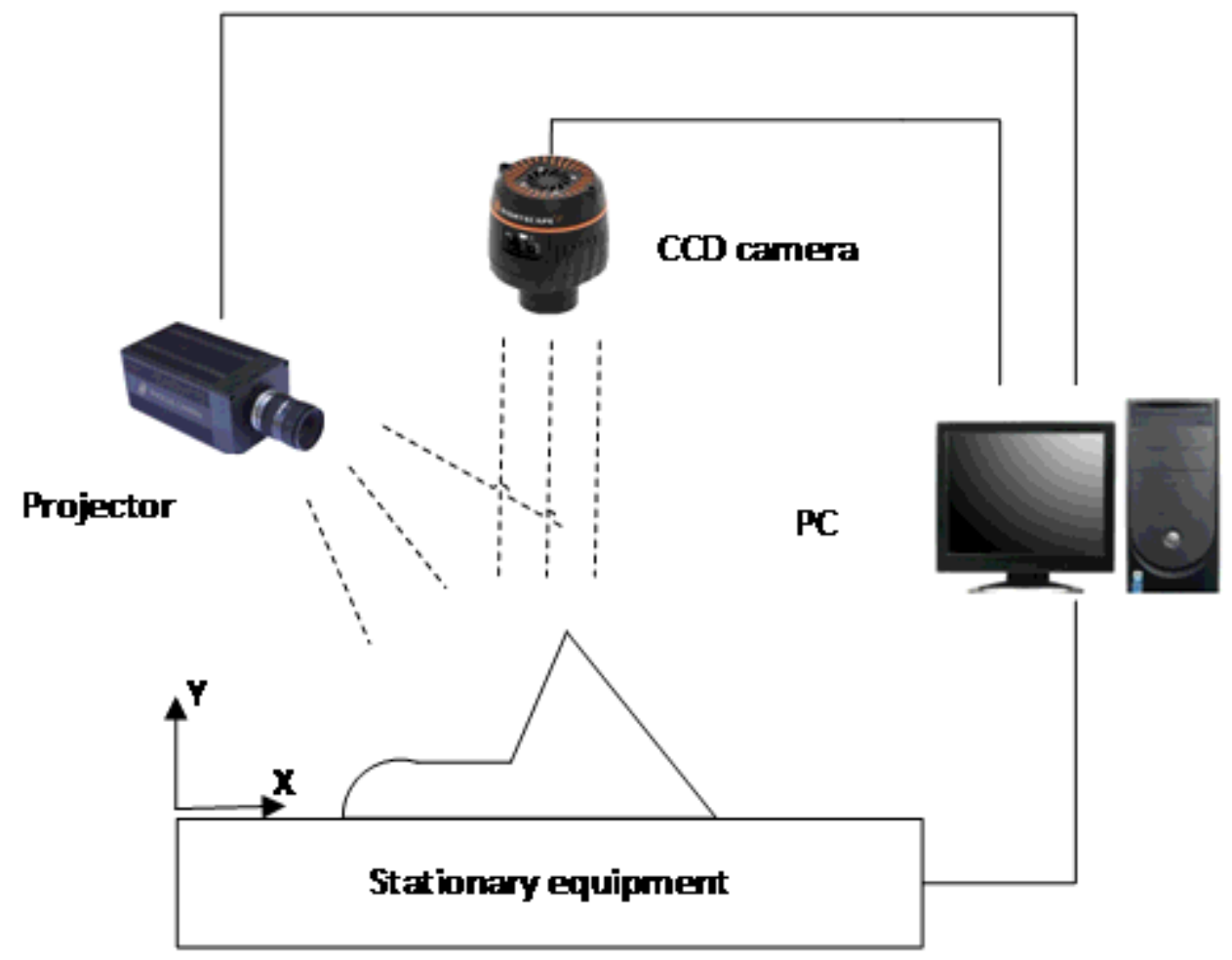

Figure 4

Test system light path diagram 


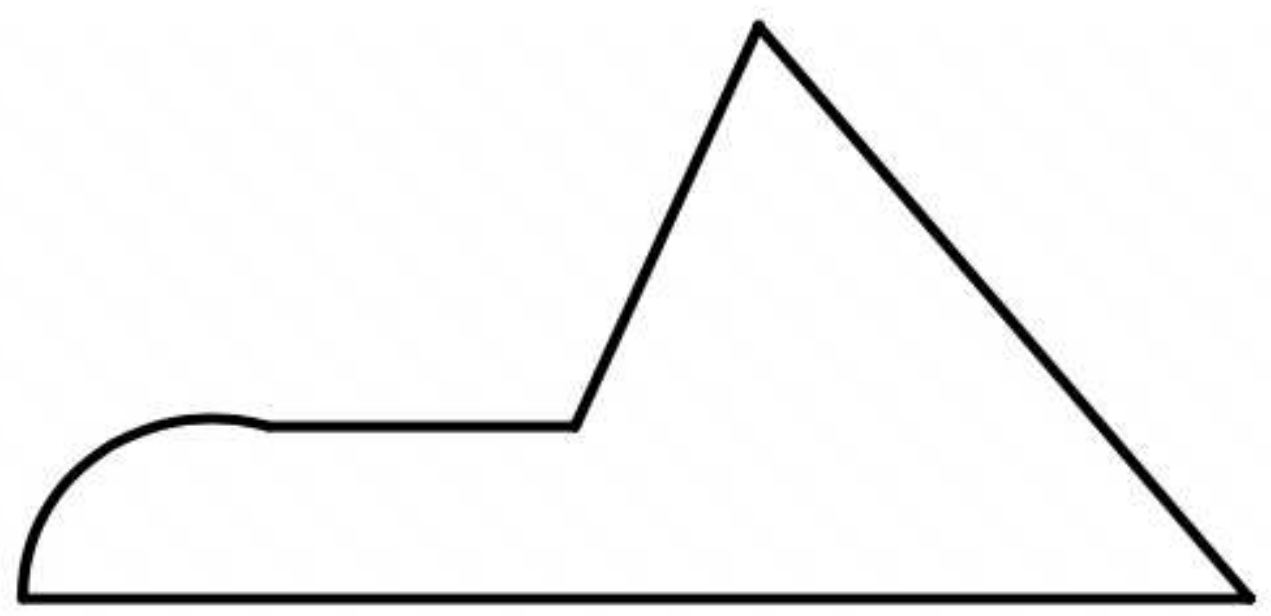

Figure 5

measured surface section
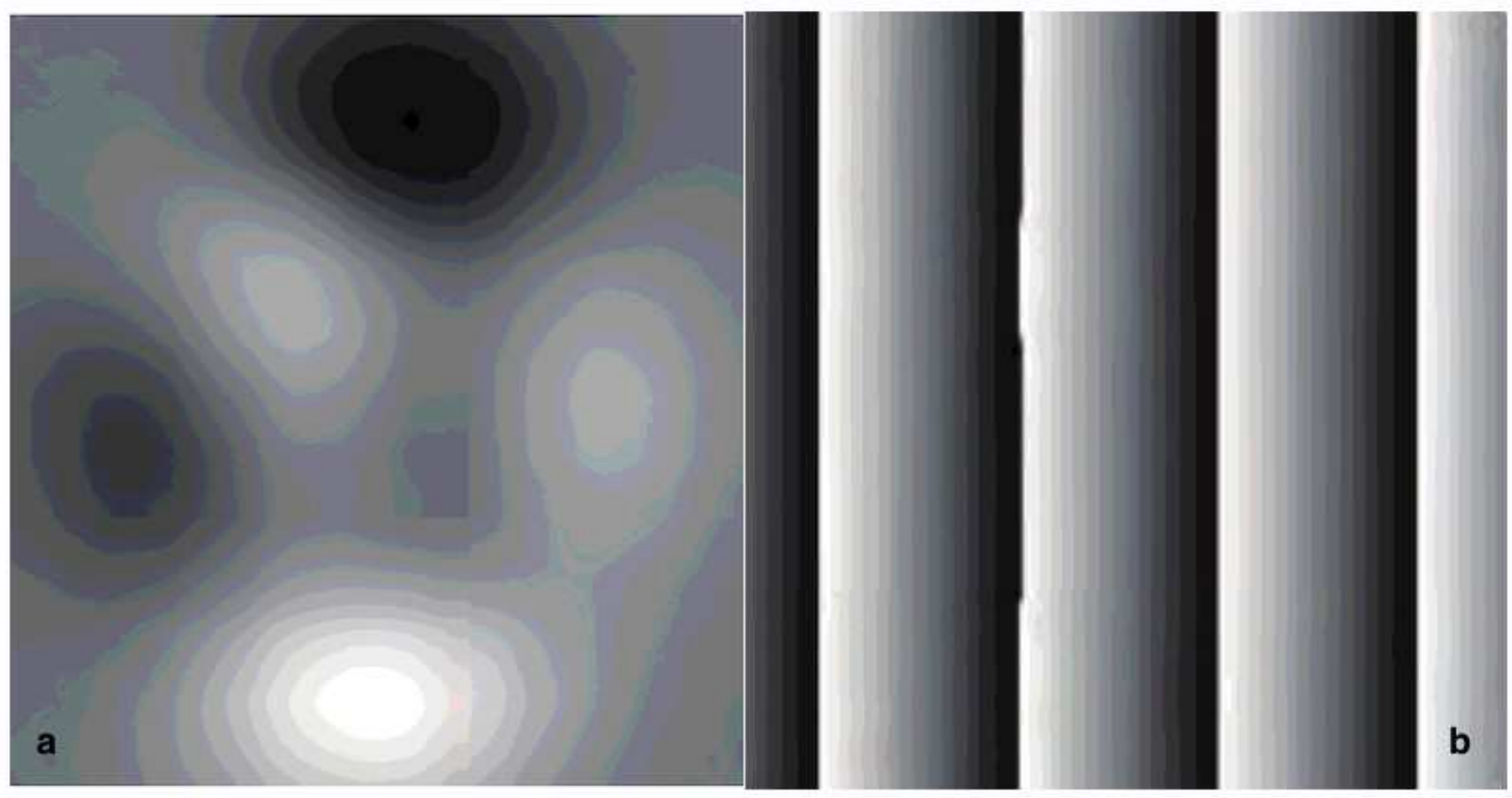

Figure 6

(a) simulated wavefront map (b) wrapped phase map 

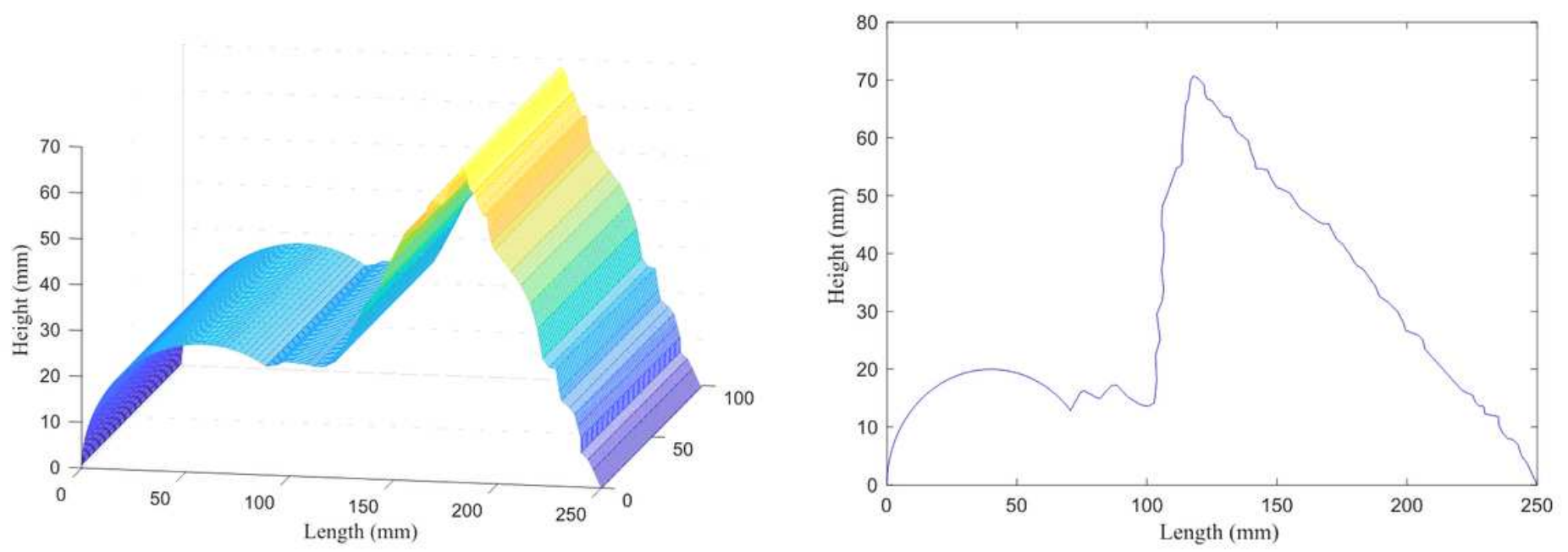

Figure 7

Results of the iterative algorithm measurement. (a)The measured surface shape of the iterative method (b)The measured surface profile of the iterative method
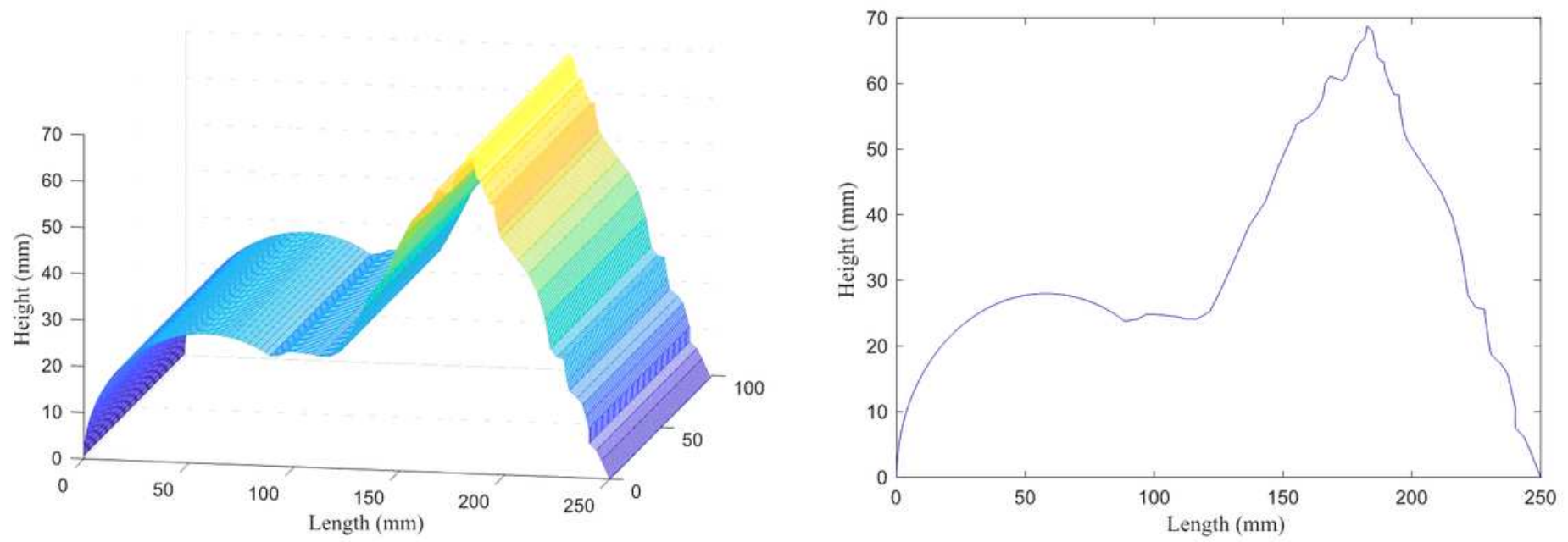

Figure 8

Results of the iterative algorithm measurement. (a)The measured surface shape of the four-step phase shift algorithm (b)The measured surface profile of the four-step phase shift algorithm 

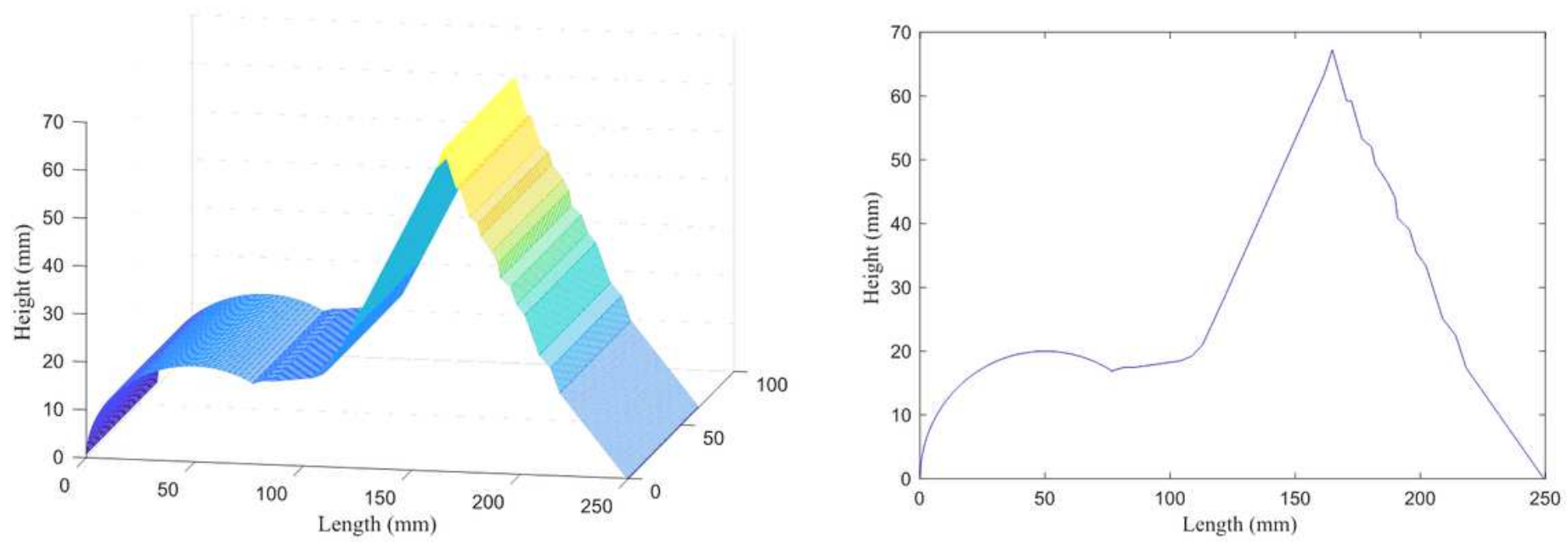

Figure 9

Results measured by a variable-phase phase shift phase extraction algorithm based on selected samples. (a)The measured shape surface solved by the variable frequency phase shift phase extraction algorithm based on the selected sampling (b)Sectional view of the measured shape solved by the variable frequency phase shift phase extraction algorithm based on the selected sampling 